\title{
Cyclization studies of Japanese encephalitis virus non-coding RNA terminal regions.
}

Tyler Mrozowich ${ }^{1}{ }^{\dagger}$, Sean M. Park ${ }^{1+}$, Maria Waldl ${ }^{2,3}$, Amy Henrickson $^{1}$, Corey R. Nelson ${ }^{1}$, Borries Demeler $^{1,4,5}$, Ivo L. Hofacker ${ }^{2,6}$, Michael T. Wolfinger ${ }^{2,6}$, Trushar R. Patel ${ }^{1,5,7,8 *}$

1. Department of Chemistry and Biochemistry, Alberta RNA Research and Training Institute, University of Lethbridge, 4401 University Drive, Lethbridge, AB T1K 3M4, Canada

2. Department of Theoretical Chemistry, University of Vienna, Währinger Strasse 17, 1090, Vienna, Austria

3. Center of Anatomy \& Cell Biology, Division of Cell \& Developmental Biology, Medical, University of Vienna, Schwarzspanierstrasse 17, 1090, Vienna, Austria

4. Department of Chemistry and Biochemistry, University of Montana, Missoula, MT 59812, USA

5. NorthWest Biophysics Consortium, University of Lethbridge, University of Lethbridge, 4401 University Drive, Lethbridge, AB T1K 3M4, Canada

6. Bioinformatics and Computational Biology, Faculty of Computer Science, University of Vienna, Währinger Strasse 29, 1090, Vienna Austria

7. Li Ka Shing Institute of Virology, University of Alberta, Edmonton, T6G 2E1, Alberta, Canada

8. Department of Microbiology, Immunology \& Infectious Diseases, Cumming School of Medicine, University of Calgary, Calgary, T2N 4N1, Canada

† These authors contributed equally to this work

*-Corresponding author email trushar.patel@uleth.ca 


\begin{abstract}
Many flaviviruses such as Dengue and West Nile virus undergo essential long-range interactions of their 5' and 3' terminal regions (TRs), mediated by a conserved complementary cyclization sequence. However, we lack insights into such long-range interactions for the Japanese Encephalitis virus (JEV). Here, we utilized an extensive, multi-faceted approach involving computational and biophysical tools. We performed multi-angle light scattering (SEC-MALS) to determine absolute molecular weights of JEV TRs, and their complex concluding they form a 1:1 complex and corroborated this interaction using analytical ultracentrifugation (AUC). The microscale thermophoresis (MST) experiments demonstrated that the $5^{\prime}$ and 3' TR of JEV interact with $\mathrm{nM}$ affinity, which is significantly reduced without the conserved cyclization sequence. To our knowlwege, this is the first study representing the application of three key biophysical methods (AUC, MST and SEC-MALS) to study RNA-RNA interactions. Furthermore, we performed computational kinetic analyses corroborating our MST studies showing the essential role of the cyclization sequence in the RNA-RNA interaction. The binding affinity of this biologically critical event is a vital pharmacological feature that can influence potential competitive inhibition by therapeutics. This evidence can also influence pharmaceutical interventions aimed at inhibiting the conserved flavivirus cyclization, thus, interrupting replication across the flavivirus family.
\end{abstract}




\section{Introduction}

Japanese Encephalitis virus (JEV) is a mosquito-borne flavivirus in the genus Flavivirus (family Flaviviridae), which contains several pathogenic viruses such as Dengue virus (DENV), West Nile virus (WNV), Zika virus (ZIKV), and yellow fever virus (YFV).JEV is the leading cause of viral encephalitis in Southeast Asia and the Western Pacific, with approximately 68,000 cases globally each year (1). Infection by JEV can present as asymptomatic or symptomatic, with the latter having a fatality rate of $20-30 \%$, with an additional $20-30 \%$ resulting in permanent neurological sequelae such as paralysis, recurrent seizures, or the inability to speak $(1,2)$. Currently, there are no approved treatments available following infection by JEV or other flaviviral infections (3), which lends to the importance of further investigation. JEV is transmitted through competent mosquito vectors of the genera Aedes and Culex (4), suggesting that flaviviral infections will likely become more prevalent as global temperatures rise and vector populations expand (5).

JEV, like other flaviviruses, is an enveloped virus with a single-stranded (+)-sense RNA genome of approximately $11,000 \mathrm{nt}$ in length (6). A single open reading frame, which encodes for a polyprotein, is flanked by highly structured $5^{\prime}$ and $3^{\prime}$ untranslated regions (UTRs) (7). The genome has a type I cap at the $5^{\prime}$ end (m7GpppAMP) and lacks polyadenylation at the $3^{\prime}$-terminus. The single open reading frame is cleaved post-translationally into three structural proteins and seven non-structural proteins $(8,9)$. During replication, the $5^{\prime}$ and $3^{\prime}$ terminal regions (TRs) in flaviviruses undergo long-range intragenomic RNA-RNA interactions, thereby forming a so-called panhandle structure that mediates recruitment of the viral RNAdependent RNA polymerase (NS5) (10). Removal of the TRs has shown inhibition of viral replication (1013). A cyclization sequence of 11 nt is complementary in both the $5^{\prime}$ and $3^{\prime}$ TRs, facilitating this interaction (14). Furthermore, these terminal regions show binding with a variety of human host proteins (15), including but not limited to numerous DEAD-box helicases (16).

In WNV and DENV, the 5'-3' long-range interaction has been previously demonstrated (12$14,17,18)$. WNV mutants unable to undergo genomic cyclization were non-replicative, while restorative mutations returned replicative capabilities (12). In addition, 5'-3' complementarity has shown a functional role in replicating other flaviviruses such as Kunjin virus, ZIKV, and YFV (19-23). Genome cyclization in JEV has been computationally predicted previously (24); however, detailed experimental verification is still missing. As a critical step in viral replication, and due to the absence of current treatments, investigation of this viral cyclization should be considered a potential therapeutic avenue for JEV and other flaviviruses.

In this study, we sought to explore flavivirus cyclization in a virus species that has not been studied for this aspect of the viral life cycle previously. We identified an isolate of JEV that we hypothesized to interact with high affinity through bioinformatics analysis. Subsequently, we computationally evaluated a consensus duplex structure of the $5^{\prime}$ and $3^{\prime}$ TRs of 20 different flaviviruses and measured their conservation. With this knowledge and utilizing various biophysical characterization techniques, we directly demonstrate for the first time that JEV 5'-3' TRs interact in vitro with nanomolar affinity and with 1:1 stoichiometry. Furthermore, we isolated and identified the RNA-RNA complex using size exclusion coupled multi-angle laser light scattering (SEC-MALS). We additionally use analytical ultracentrifugation (AUC) as an orthogonal biophysical validation as evidence of JEV 5'-3'TR interaction. Finally, we show computational evidence which complements our experimental data showing that the cyclization sequence interaction is kinetically favorable and will out-compete potential homo-dimer RNA interactions. In summary, our study provides the first direct evidence that JEV TRs can cyclize in vitro. We 
bioRxiv preprint doi: https://doi.org/10.1101/2022.02.01.478553; this version posted February 2, 2022. The copyright holder for this preprint

(which was not certified by peer review) is the author/funder, who has granted bioRxiv a license to display the preprint in perpetuity. It is made available under aCC-BY-ND 4.0 International license.

also show that the genus Flavivirus has a high degree of sequence conservation of the cyclization sequence and the resulting duplex structure. This characterization can be used as a foundation in potential pharmaceutical therapies to inhibit viral replication through cyclization interruption. 


\section{Materials and Methods}

\section{Computational assessment of genome cyclization}

Putative interaction sites between $3^{\prime}$ and 5'TRs of 109 JEV isolates were predicted with IntaRNA v3.2.0, RNAup v2.4.18, and RNAcofold (25-29). A consensus secondary structure of the $5^{\prime} / 3^{\prime}$ terminal regions in 20 phylogenetically related flaviviruses was computed with RNAalifold v2.4.18 (30) from the ViennaRNA Package v2.4.18 (31), based on a structural nucleotide multiple sequence alignment computed with LOCARNA v2.0.0RC8 (32).

We evaluated whether the predicted TR interactions are kinetically feasible by applying a novel direct path model. In this model, the full target interaction is formed starting from an initial seed interaction, which folds into the target interaction by adding or removing target base pairs. All substructures on a folding path consist of consecutive base pairs of the target interaction. Consequently, paths are 'direct' since they cannot contain detours via non-target interaction base pairs. We compute the free energy of each substructure analogous to the RNAup energy model. The two main contributions are the cost of making the interaction site unpaired in the two intramolecular structures and the stabilizing contribution of the interaction base pairs. Both contributions were obtained from the ViennaRNA library. We can model interaction formation as a Markov process based on these direct paths model and the energy model/function. The energy barrier along the direct folding path (activation energy) determines the overall rate at which an interaction is formed. Since the model's possible substructures (aka states) can be completely described by their first base pair i and last base pair j, they form a 2D energy landscape, and any direct path from a first base pair to the full interaction can be drawn as shown in figure S2. Plots of these energy landscapes allow for the visual identification of barriers along the folding path.

\section{Preparation and Purification of non-coding RNA}

JEV 3'TR and 5'TR constructs of JEV were designed based on the GenBank sequence of KR265316.1, while JEV 5' and $3^{\prime}$ UTR were based on KT957419.1. CDNA sequences were prepared in pUC57 plasmids under the control of T7 RNA polymerase. The cDNA was flanked by two additional G nucleotides on the $5^{\prime}$ end and an Xbal restriction enzyme cut site (T^CTAGA) on the $3^{\prime}$ end. Each RNA construct is graphically represented in figure 1 to display clarity on which portion of the terminal region is involved in each

\begin{tabular}{|c|c|c|c|c|}
\hline Name & Genomic Location & Length (NTs) & Sequence & Mw (kDa) \\
\hline JEV 5' TR & $1-224$ & 228 & $\begin{array}{l}\text { 5'GGAGAAGUUUAUCUGUGUGAACUUCUUGGCUUAGUAUCGUUGAGAAGAAUCGAGAGAUU } \\
\text { AGUGCAGUUUAAACAGUUUUUUAGAACGGAAGAUAACCAUGACUAAAAAACCAGGAGGGCC } \\
\text { CGGUAAAAACCGGGCUAUCAAUAUGCUGAAACGCGGCUUACCCCGCGUAUUCCCACUAGUGG } \\
\text { GAGUGAAGAGGGUAGUAAUGAGCUUGUUGGACGGCAGAGGGCCAGU3' }\end{array}$ & 73.9 \\
\hline JEV 5' UTR & $1-96$ & 100 & $\begin{array}{l}\text { 5'GGAGAAGUUUUAUCGUGUGAACUUCUUGGCUUAGUAUCGUUGAGAAGAAUCGAGAGAUU } \\
\text { AGUGCAGUUUAAACAGUUUUUUAGAACGGAAGAACAACCU3' }\end{array}$ & 32.2 \\
\hline JEV 3' TR & 10758-10976 & 221 & $\begin{array}{l}\text { 5'GGUUAGAGGAGACCCCGUGGAAACAACAACAUGCGGCCCAAGCCCCCUCGAAGCUGUAUAG } \\
\text { GAGGUGGAAGGACUAGAGGUUAGAGGAGACCCCGCAUUUGCAUCAAACAGCAUAUUGACAC } \\
\text { CUGGGAAUAGACUAGGAGAUCUUCUGCUCUAUCUCAACAUCACUUACUAGGCACAUAUCGCC } \\
\text { GAAGUAUGUAGCUGGUGGUAGGAAGAACACACGAUCU3' }\end{array}$ & 71.4 \\
\hline JEV 3' UTR & $10395-10965$ & 573 & $\begin{array}{l}\text { 5'GGACAGGAUAAAGUCAUAUGUGUAAUGUGAGAUAAGAAAAUGUGCAUGUGGAGUCAGGC } \\
\text { CAGCAAAAGCUGCCACCGGAUACUGAGUAGACGGUGCUGCCUGCGUCUCAGUCCCAGGAGGA } \\
\text { CUGGGUUAACAAAUCUGACAACGGAAGGUGGGAAACCCUCAGAACCGUCUCGGAAGCAGG } \\
\text { UCCCUGCUCACCGGAAGUUGAAAGACCAACGUCAGGCCACAAUUCUGUGCCACUCCGCUGGG } \\
\text { GAGUGCGGCCUGCGCAGCCCCAGGAGACUGGGUUACAAAGCCGUUGAGGCCCCACGGCC } \\
\text { CAAGCCUCGUCUAAGAUGCAAUAGACUAGGUGUAAGGACUAGAGGUUAGAGGAGACCCGU } \\
\text { GGAAACAACAUUGUGCGGCCCAAACCCCUCGAAGCUGUAGAGGAGGUGGAAGACUAGAG } \\
\text { GUUAGAGGAGACCCCGCAUUUGCAUCAAAACAGCAUAUUGACACCUGGGAAUAGACUGGA } \\
\text { GAUCUUCUGCUCUAUCUCAACAUCAGCUACUAGGCACAGAGCGCCGAAGUAUGUAGCUGGU } \\
\text { GGUGAGGAAGAACCAGGAUCUU3' }\end{array}$ & 185.6 \\
\hline
\end{tabular}


experiment. All RNA construct sequences are listed below, and underlined regions represent the theoretical cyclization sequence which base pairs complementary between the $5^{\prime}$ and $3^{\prime}$ TR.

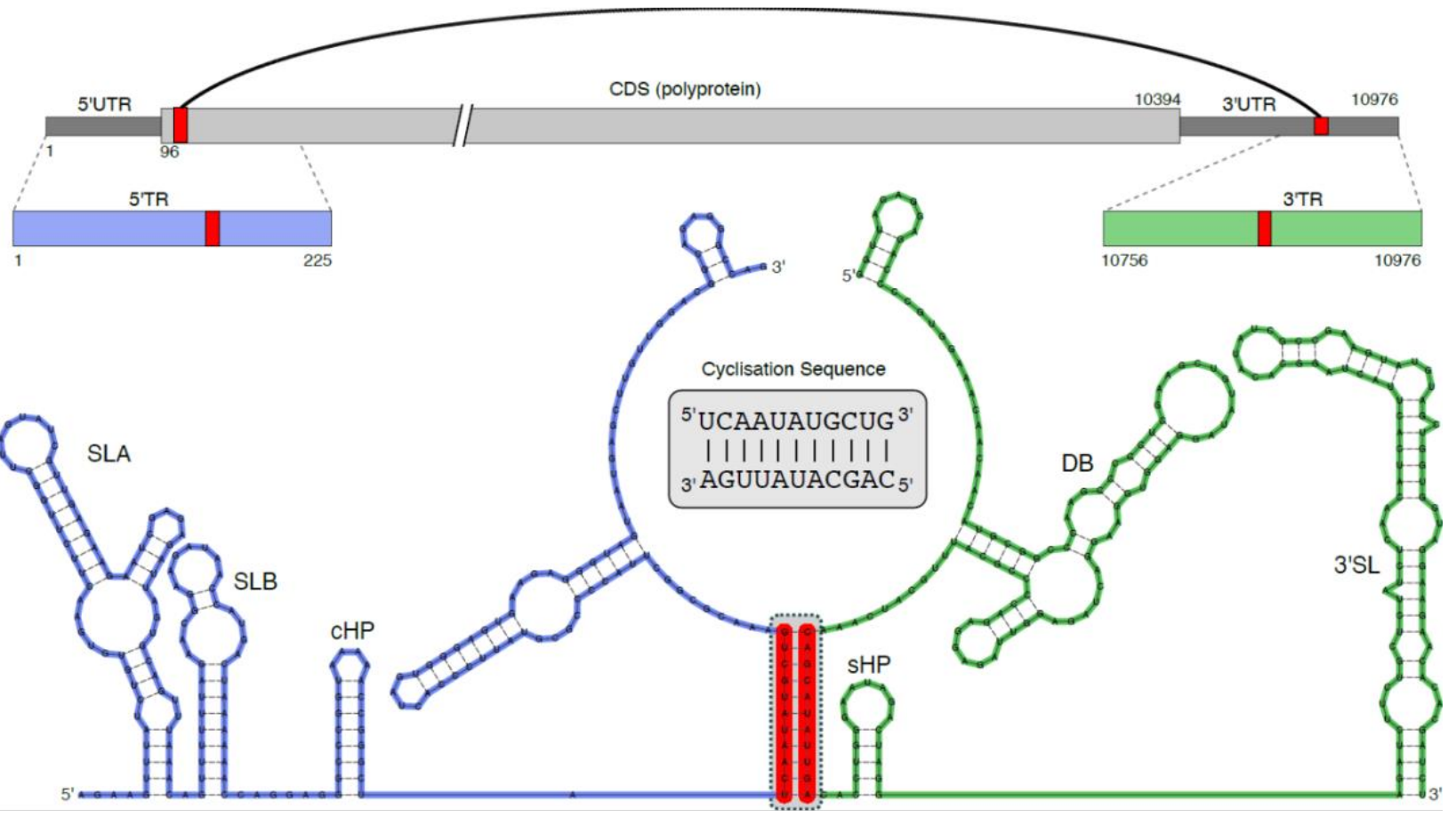

Figure 1. JEV structural organization and theoretical secondary structure prediction. Visual representation of JEV genome organization and different construct regions, which were designed and studied. Predicted secondary structure of the cyclization between JEV 5'TR and JEV 3'TR from constraining co-folding, highlighted red portions represents the 11 conserved nucleotides which form a stable base-pairing interaction to facilitate the long-range genome interaction.

RNA was prepared via in vitro transcription reaction using T7 RNA polymerase (purified in-house) followed by size-exclusion chromatography (SEC) purification using a Superdex 200 Increase GL 10/300 (Global Life Science Solutions USA LLC, Marlborough, MA, USA) in JEV RNA Buffer (10 mM Bis-tris pH 5.0, $100 \mathrm{mM}$ $\mathrm{NaCl}, 15 \mathrm{mM} \mathrm{KCl} 15 \mathrm{mM} \mathrm{MgCl}_{2}, 10 \%$ glycerol) via an ÄKTA pure FPLC (Global Life Science Solutions USA LLC, Marlborough, MA, USA) with a flow rate of $0.5 \mathrm{~mL} / \mathrm{min}$. Urea-polyacrylamide gel electrophoresis (Urea-PAGE) was utilized to analyze SEC peak fractions. We mixed $10 \mu \mathrm{L}$ of each fraction with $2 \mu \mathrm{L}$ of denaturing RNA loading dye and loaded it into a $1.0 \mathrm{~cm}$ well PAGE casting plate (Bio-Rad Laboratories, Mississauga, ON, Canada). Urea-PAGE (7.5\%) was run at room temperature, 300V, for 25 min (20 min for JEV $5^{\prime}$ TR) in 0.5x TBE (Tris-Borate-EDTA) buffer, followed by staining with Sybr safe (Thermofisher Scientific, Saint-Laurant, QC, Canada) and visualization. Fractions containing a single band were deemed acceptable and used in subsequent experiments.

\section{Light Scattering}

Multi-angle laser light scattering (MALS) experiments were performed on a Dawn ${ }^{\circledR}$ (Wyatt Technology Corporation, Santa Barbara, CA, USA) multi-angle laser light scattering instrument with 18 detector angles utilizing a $658 \mathrm{~nm}$ laser. Furthermore, an Optilab ${ }^{\circledR}$ (Wyatt Technology Corporation, Santa Barbara, CA, USA) refractometer was also positioned downstream to measure the solvent refractive index 
and absolute concentration of solutes. These instruments were positioned in line with an SEC column (Superdex 200 increase 10/300 GL, Global Life Science Solutions, USA LLC, Marlborough, MA, USA) attached to an ÄKTA pure FPLC (SEC-MALS). All experiments were performed at ambient room temperature $\left(20^{\circ} \mathrm{C}\right)$ with the same flow rate and buffer as previous SEC experiments described above. The refractive index of the solvent was defined as 1.3308 (measured by in-line Optilab ${ }^{\circledR}$ refractometer), while the $\mathrm{dn} / \mathrm{dc}$ (refractive index increment) value of $0.1720 \mathrm{~mL} / \mathrm{g}$ was used for all RNAs (33). The final concentration of JEV 3' TR and 5' TR RNA used were $1.3 \mu \mathrm{M}$ and $3.5 \mu \mathrm{M}$, respectively, in a combined volume of $500 \mu \mathrm{L}$. Samples were incubated for 3 hours prior to loading. Data were analyzed using Astra v8.0.0.25, and absolute molecular weight $\left(M_{w}\right)$ was calculated using Equation 1 for each elution point, where: $R(\theta)$ is Rayleigh's ratio, $K$ is the polymer constant, and $c$ is the concentration of the solution.

\section{Equation 1}

$$
M_{w}=\frac{R(\theta)}{K^{*} c}
$$

\section{Analytical Ultracentrifugation}

AUC sedimentation velocity data for JEV 5' TR and 3' TR were collected using a Beckman Optima AUC centrifuge and an AN50-Ti rotor at $20^{\circ} \mathrm{C}$. We loaded JEV 5'TR (214 nM, 0.5 OD @260 nm), JEV 3'TR (224 nM, 0.5 OD @260 nm), and a 1:1 mixture of JEV 5'TR and 3'TR samples into Epon-2 channel centerpieces in JEV RNA Buffer. We centrifuged samples at 25,000 revolutions per minute and collected scans at 20-second intervals. We used the UltraScan-III package (34) to analyze all data via supercomputer calculations in-house. We analyzed the SV-AUC data using two-dimensional spectrum analysis (2DSA) with simultaneous removal of time-invariant noise, meniscus, and bottom positions fitted, followed by enhanced van Holde-Weischet analysis (35). We estimated the buffer density and viscosity corrections with UltraScan $(1.0269 \mathrm{~g} / \mathrm{cm} 3$ and $1.293 \mathrm{cP}$, respectively). All hydrodynamic parameters were corrected to standard conditions at $20^{\circ} \mathrm{C}$ and water.

\section{Fluorescent labeling of RNA}

Purified RNA was subjected to labeling at the 5' end by the fluorophore Alexa 488 (Thermofisher Scientific, Saint-Laurant, QC, Canada). One milligram of A488 was resuspended in $175 \mu \mathrm{L}$ of $0.2 \mathrm{M} \mathrm{KCl} .7 .5 \mu \mathrm{L}$ concentrated RNA $(>100 \mu \mathrm{M})$ was added to 1.25mg 1-ethyl-3-(3-dimethylamino) propyl carbodiimide hydrochloride (EDC) prior to the addition of $10 \mu \mathrm{L}$ resuspended A488. Samples were vortexed until contents were dissolved entirely before the addition of $20 \mu \mathrm{L} 0.1 \mathrm{M}$ imidazole, $\mathrm{pH} 6$. Reactions were incubated in a $37^{\circ} \mathrm{C}$ water bath overnight in the absence of light, followed by removal of free dye through 10 kDa Vivaspin ${ }^{\circledast} 500$ centrifugal concentrators (Sartorius Stedim Biotech, Göttingen, Lower Saxony, Germany). After removing all free dye, labeled RNA was diluted in JEV RNA Buffer, and fluorescence checks were conducted using microscale thermophoresis (MST).

\section{Microscale thermophoresis}

A 2-fold serial dilution was performed on the RNA ligand, either JEV 3'TR or JEV 3'UTR, where the highest concentration in the assay was $21 \mu \mathrm{M}$ and $5.5 \mu \mathrm{M}$, respectively. A constant amount of fluorescently labeled RNA Target, JEV 5'TR or JEV 5'UTR, was added to each serial dilution of RNA ligand resulting in a final concentration of $25 \mathrm{nM}$ and $52 \mathrm{nM}$, respectively. Mixtures were incubated at room temperature for 3 hours and then loaded into a Nanotemper Technologies Monolith ${ }^{\circledR}$ NT.115 instrument 
(Munich, Germany) using standard capillaries. Thermophoresis was measured at room temperature $\left(22^{\circ} \mathrm{C}\right)$ and performed using $100 \%$ excitation power along with medium IR-Laser power. Initial fluorescence migration was measured from (-1.0 to $0 \mathrm{~s}$ ) and used to normalize the measured fluorescent migration time (4.0 to $5.0 \mathrm{~s})$. Three independent replicates were analyzed using MO.Affinity Analysis software v2.1.3 and fit to the standard $K_{D}$ fit model, which describes a 1:1 stoichiometric molecular interaction, according to the law of mass action. The dissociation constant $\left(\mathrm{K}_{\mathrm{D}}\right)$ is estimated by fitting equation 1., where $F(c)$ is the fraction bound at a given ligand concentration $c$. Unbound is the $F_{\text {norm }}$ signal of the isolated target; Bound is the $F_{\text {norm }}$ signal of the complex, while $c_{\text {target }}$ is the final concentration of the target in the specific assay.

Equation 2.

$F(c)=$ Unbound $+($ Bound - Unbound $) \times \frac{c+c_{\text {target }}+K_{d^{-}} \sqrt{\left(c+c_{\text {target }}+K_{d}\right)^{2}}-4 c c_{\text {target }}}{2 c_{\text {target }}}$

\section{Results and Discussion}

\section{Computational analysis of the cyclization element in JEV and related flaviviruses}

We performed computational analysis of the potential long-range interaction between the $5^{\prime}$ and $3^{\prime}$ TRs in more than $100 \mathrm{JEV}$ isolates. This work revealed that all isolates interact via the canonical 11nt cyclization sequences present in their $5^{\prime}$ and $3^{\prime}$ TRs. Based on this work, we selected JEV isolate KR265316.1 as a model to assess long-range interactions in silico and in vitro. The Flaviviral TRs are known to harbor functional RNA elements such as stem-loops A and B in the 5'UTR, a short conserved hairpin (cHP) at the beginning of the coding region, and multiple cis-regulatory elements in the $3^{\prime} U T R$, such as dumbbell and terminal $3^{\prime}$ stem-loop $\left(3^{\prime} \mathrm{SL}\right)$ structures $(36,37)$. These conserved elements exert crucial roles in the flaviviral life cycle; therefore, we required them to be formed by constraint co-folding of the JEV $5^{\prime}$ and 3 'TRs. As presented in Figure 1, the resulting duplex structure suggests that the canonical 11nt cyclization sequence present in the $5^{\prime}$ and $3^{\prime}$ TRs is responsible for mediating the interaction. Furthermore, the other canonical secondary structures, such as SLA, SLB, and CHP in the $5^{\prime}$ TR with SHP, the $3^{\prime}$ SL, and the $3^{\prime}$ DB in the $3^{\prime}$ TR, are also present (Figure 1).

To further assess flaviviral long-range RNA-RNA interactions, we performed a comparative genomics assay in phylogenetically related viruses. To this end, we analyzed the propensity of duplex formation between 5' and 3'TRs in 20 mosquito-borne flaviviruses (Figure S2) utilizing consensus structure evaluation of the terminal genomic regions (Figure 2). Interestingly, the tendency to form a long-range interaction via the canonical cyclization motif is more pronounced in the consensus duplex than in the single sequence (JEV only) analysis. Specifically, the $11 \mathrm{bp}$ duplex is formed in the consensus structure without any constraints on the known 5' and 3' UTR elements (Figure 2). This tendency and conservation for long-range interaction in 20 sampled mosquito-borne flaviviruses reveal the importance of further understanding the cyclization interaction.

Additionally, a kinetic analysis of the canonical cyclization structure suggests that it is also kinetically favoured in all investigated JEV isolates. Exemplarily, the energy landscape of the cyclization structure from isolate KR265316.1 is shown in Figure 6 (discussed in detail later). Independent of the selected start base pair, every interaction extension step leads to a more stable structure. Thus, there are 
no barriers along the folding paths, and the interaction can form fast. We then sought to validate the in silico-derived cyclization interaction in JEV through extensive biophysical characterization in vitro.

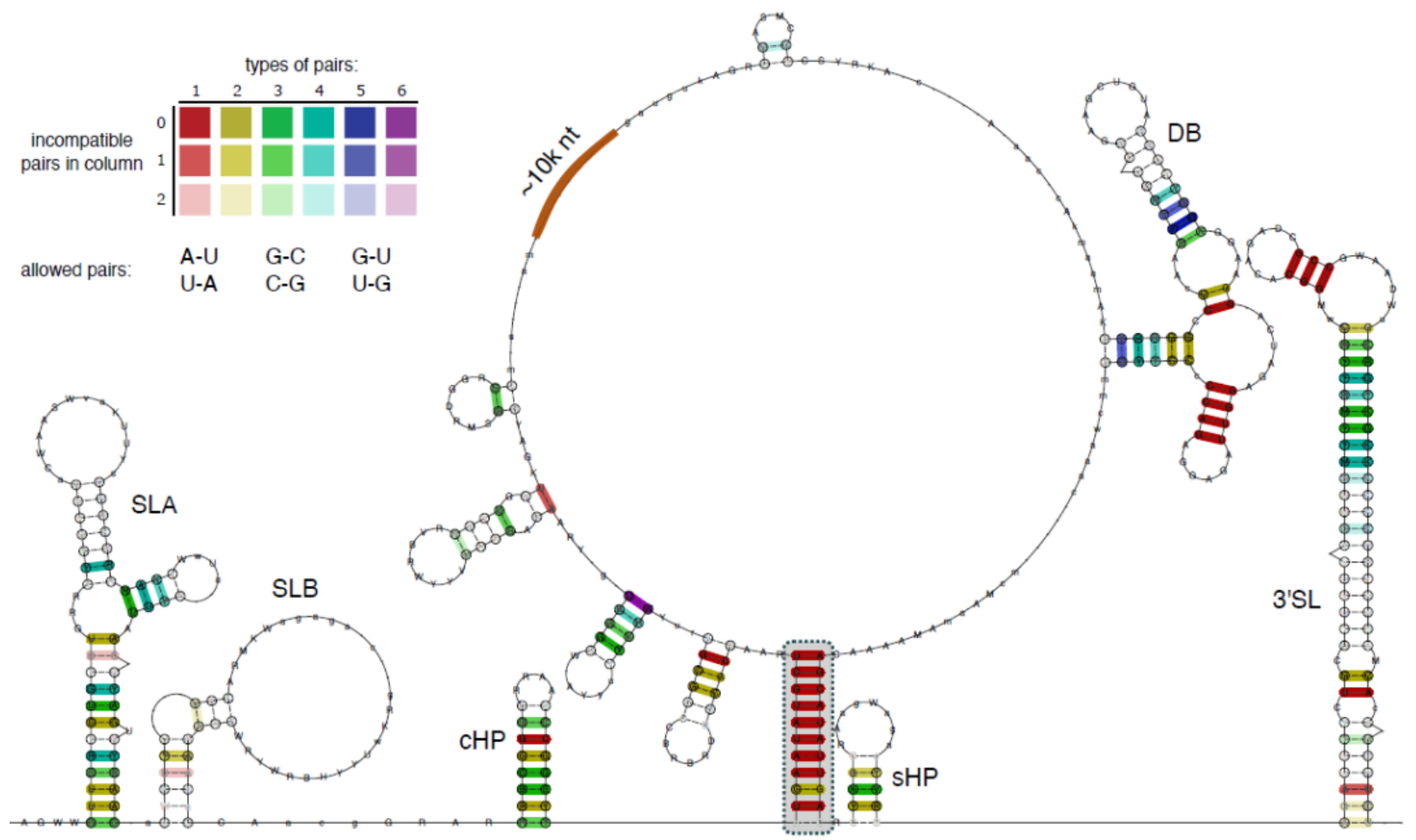

Figure 2. Consensus secondary structure of the $5^{\prime} T R / 3^{\prime} T R$ long-range interaction, computed from a structural multiple sequence alignment of 20 mosquito-borne flaviviruses. (Figure S1). Coloring of base pairs follows the RNAalifold schema and indicates different covariation levels, ranging from red (no covariation, complete primary sequence conservation) to violet (full covariation, all six possible combinations of base pairs) at corresponding columns of the underlying alignment. The duplex formed by the almost entirely sequence-conserved $11 \mathrm{nt}$ cyclization sequences (highlighted in gray) represents the only long-range interaction in the consensus structure. Canonical 5'TR elements (SLA, SLB, and CHP), as well as $3^{\prime}$ TR elements (DB, SHP, and $3^{\prime} \mathrm{SL}$ ), are predicted to fold in the consensus structure, indicating that they are energetically more favourable than an extended long-range interaction duplex structure.

\section{Purification of JEV RNA Constructs}

JEV viral non-coding RNAs were purified immediately after in vitro transcription using SEC, similar to previous works $(16,38,39)$. The elution profile for JEV 5'TR (Figure 3A) indicates that the RNA elutes at approximately $11.5 \mathrm{~mL}$ as a single monodispersed species, evident by the typical Gaussian distribution. JEV 3'TR elutes as a multimodal distribution with an observable peak at $\sim 12 \mathrm{~mL}$, consistent with its size compared to JEV 5'TR (Figure 1A). The 5'TR and 3'TR transcripts have very similar molecular weights, 73.5 $\mathrm{kDa}$ and $71.3 \mathrm{kDa}$, respectively, which is reflected in the chromatogram as both monomeric peaks appear to have a similar elution volume $\sim 11.5-12.0 \mathrm{~mL}$ (Figure 3A). Higher-order oligomeric species for JEV 3'TR can be observed at $\sim 8-11 \mathrm{~mL}$, and these elution fractions we avoided for downstream experiments. To determine if the SEC elution fractions contained the correct-sized RNA species, we utilized urea-PAGE. 
Figure 3B (left side) shows JEV 3'TR fractions from the right side of the $\sim 12 \mathrm{~mL}$ peak containing the appropriate length RNA species (227 nt). Only selected fractions $(12.5-13.5 \mathrm{~mL})$ were pooled to avoid contamination from any oligomeric species (Figure 3A, red). JEV 5'TR elution fractions (Figure 3B, right side) demonstrate, as expected, a single species of the correct RNA size (221 nt) across the peak. These fractions were pooled similarly to JEV 3'TR and highlighted for clarification (Figure 3A, green). JEV 3'UTR and $5^{\prime}$ UTR (controls used later) were transcribed and purified identically to the above RNA, and only fractions containing a single band were pooled and used in downstream experiments.

A

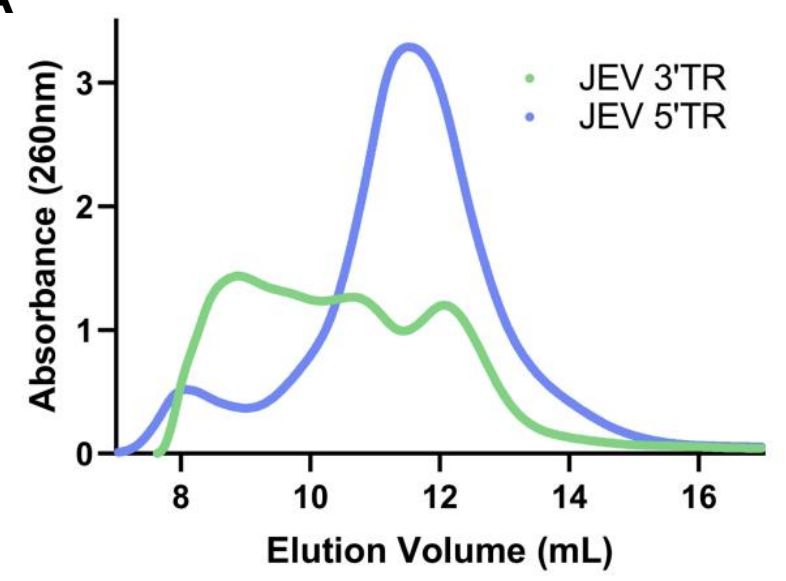

B

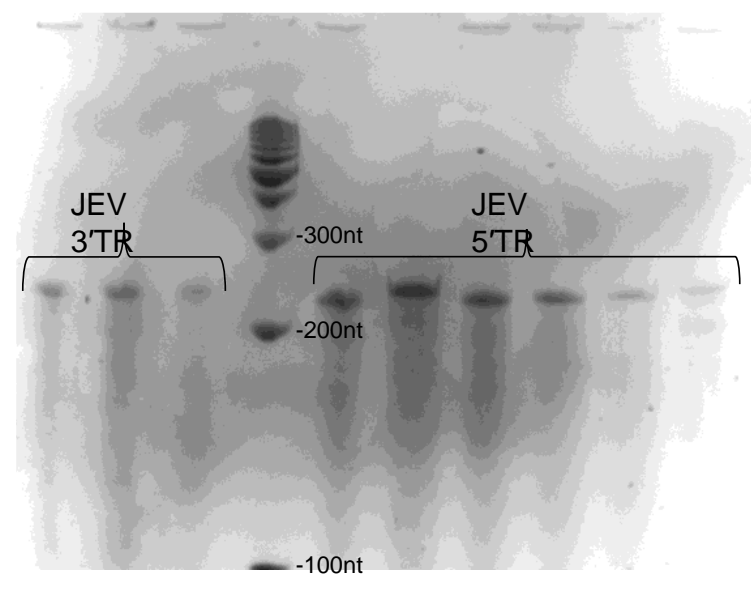

Figure 3. Purification of JEV TR RNA A) Size Exclusion Chromatogram representing the purification of both RNA. Shaded boxes represent the region collected for downstream experiments to avoid any potential oligomeric species. B) Urea PAGE of associated size exclusion chromatography fractions showing a single size of RNA ( 220nt), which is the correct size of the expected RNA.

\section{Biophysical Analysis of JEV 5' and 3'TR Complex}

Due to the highly conserved nature of Flavivirus TRs, it is theorized that JEV will utilize similar $3^{\prime}-$ 5' long-range TR interactions as a necessary step in viral RNA amplification, like other members (49). This interaction has been shown in various Flaviviridae family members, such as Dengue and West Nile Virus $(12-14,17,18)$. SEC can separate biomolecules based on their sizes, and MALS allows for the absolute molecular weight determination of biomacromolecules in solution (40); we utilized SEC-MALS to investigate if the $5^{\prime}$ and $3^{\prime}$ TRs form a complex. Figure 4A represents the elution profiles of JEV $3^{\prime} T R$, which indicates that it is almost entirely monodispersed eluting at $\sim 12 \mathrm{~mL}$ with a minor peak of potential oligomeric assembly at $\sim 11.5 \mathrm{~mL}$. The elution profile of $3^{\prime} \mathrm{TR}$ is also consistent with our initial purification using SEC, where JEV 3'TR oligomerizes into higher-order species. JEV 5'TR also elutes at $\sim 12 \mathrm{~mL}$, consistent with previous purifications; however, it also contains a shoulder at $11.5 \mathrm{~mL}$ (Figure 4A).

We then mixed both JEV 3'TR and 5'TR and performed SEC-MALS experiments. The RNA-RNA mix resulted in a bimodal SEC chromatogram distribution, with an additional peak eluting at $\sim 10.5 \mathrm{~mL}$ (Figure $4 \mathrm{~A}$, purple). Furthermore, we see a peak at $\sim 12 \mathrm{~mL}$ where excess JEV 5'TR elutes as a monomer, consistent 
with the concentrations we mixed ( $3.5 \mu \mathrm{M}$ of JEV 5'TR vs. $1.3 \mu \mathrm{M}$ of JEV $\left.3^{\prime} T R\right)$. These results indicate that the TR complex has a different elution profile upon binding than any oligomeric assembly or monomeric species. Successful separation of the JEV TR RNA complex allowed us to calculate the absolute molecular weight of each species. As presented in Figure 4B, the SEC-MALS-derived molecular weights are consistent across both peaks, indicating that both are homogenous species. Excess unbound monomer(s) elute as a single peak and are characterized at $\sim 75 \mathrm{kDa}$, which is expected considering the similarity in their theoretical weights (73.9 kDa \& $71.4 \mathrm{kDa})$. The molecular weight of the complex ( 150kDa) is double that of each TR in isolation ( 75kDa) and very similar to the predicted molecular weight of the complex (145.3 $\mathrm{kDa})$. This change in molecular weight is indicative of a 1:1 stoichiometric interaction. This stoichiometric determination is vital for biological relevance, considering these terminal regions exist on the same genome and come together to cyclize.

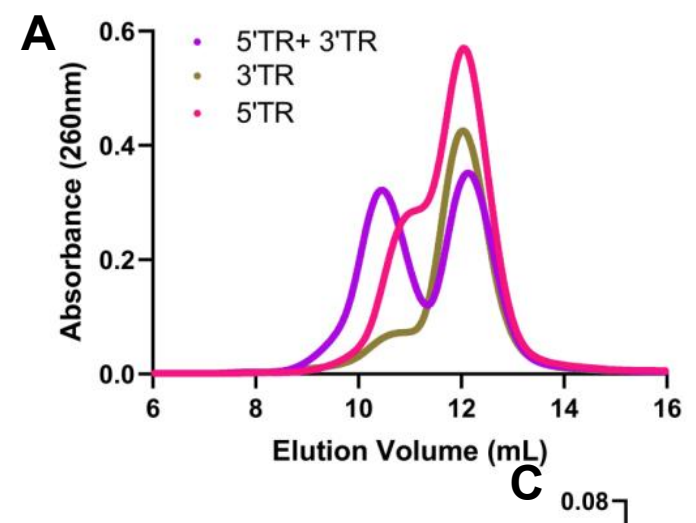

B
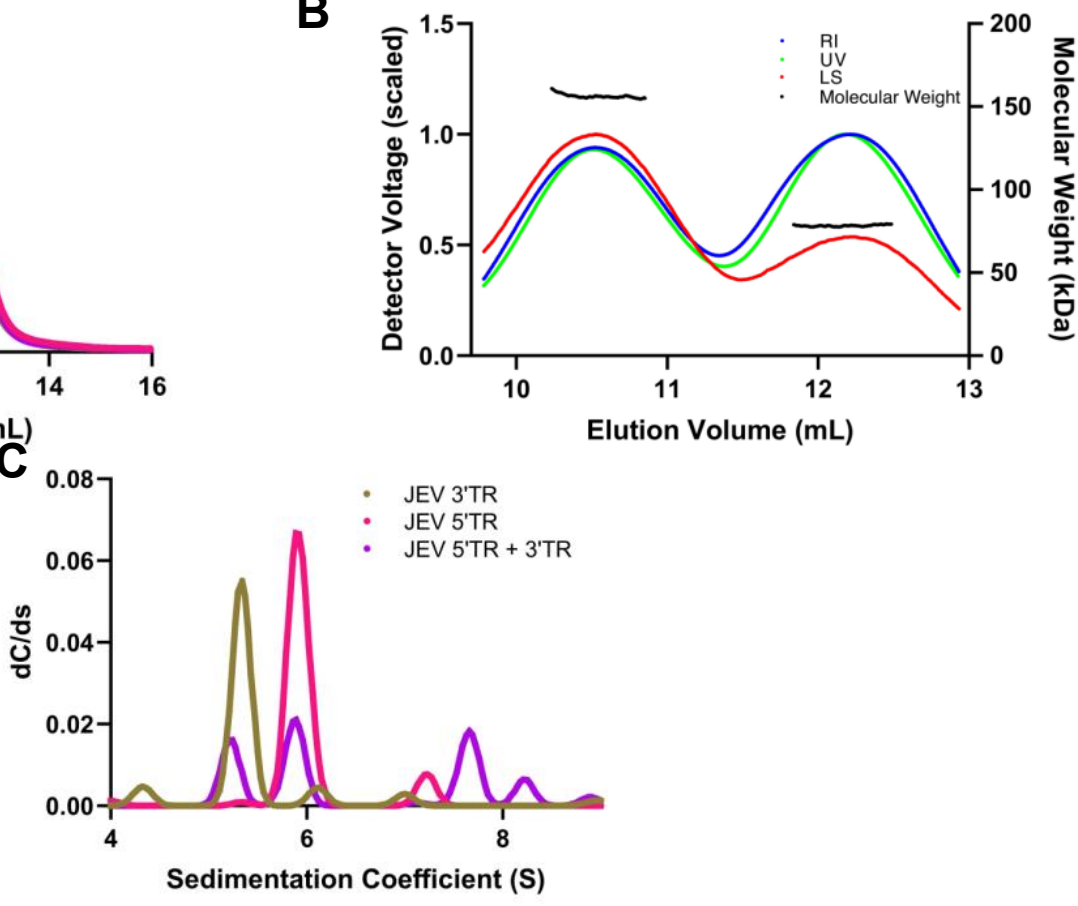

Figure 4. Light scattering analysis of JEV TR RNA cyclization A) Multiple size exclusion chromatography profiles associated with SEC-MALS. B) MALS traces of each peak from the $5^{\prime} T R+3^{\prime} T R$ run and the absolute molecular weight across them. C) Sedimentation distribution profiles of JEV 5' TR and 3'TR obtained from sedimentation velocity-analytical ultracentrifugation. Sedimentation coefficient values are corrected to standard solvent conditions $\left(20^{\circ} \mathrm{C}\right.$, water $)$

AUC is a powerful biophysical technique often used to study the purity of biomolecules in solution (41). AUC subjects biomolecules to extremely high centrifugal force (up to $250,000 \times \mathrm{g}$ ), separating them based on size, anisotropy, and density while monitoring sedimentation via an optical system. While SECMALS can provide us with absolute molecular weight determination, since both JEV 5'TR and 3'TR are of 
similar molecular weight, we needed further validation to rule out potential RNA self-oligomerization as an explanation of the 5'TR-3'TR peak in figure 3A. Therefore, we utilized the specialized capabilities of AUC to provide us with an orthogonal technique that could validate our SEC-MALS results. While both JEV 5'TR and 3'TR are similar in length, the sequences differ significantly, which will cause a change in secondary structure and, ultimately, tertiary structure. If these potential tertiary structural differences are significant enough, there should be a difference between the JEV 5'TR and 3'TR sedimentation. Both JEV 5'TR and 3'TR show highly monodispersed sedimentation profiles, evident by a single Gaussian distribution. JEV 5'TR is represented by a primary sedimentation profile at $\sim 5.9 \mathrm{~S}$, while JEV $3^{\prime}$ TR is represented at $\sim 5.3 \mathrm{~S}$ (Figure $4 \mathrm{C}$ ). These distributions confirm that not only are the JEV TRs homogenous, but they are also likely sediment differently based upon tertiary structure differences. This difference in sedimentation profiles allowed us to validate our previous SEC-MALS results by mixing JEV 5'TR and 3'TR in a 1:1 ratio and performing another sedimentation velocity experiment. As with previous SEC-MALS experiments, the AUC results show an additional sedimentation peak at $\sim 7.6 \mathrm{~S}$, which is not present in the individual TR experiments allowing us to confirm JEV 5'-3' TR interaction (Figure 4C). Additionally, since the concentrations of both RNA were $\sim 200 \mathrm{nM}$, we can also conclude that the binding affinity $\left(K_{D}\right)$ is likely in the low nanomolar range. We believe that this experiment provides the first evidence of an RNA-RNA interaction via AUC. Taking both the AUC and SEC-MALS results together, we can confidently conclude that the additional peak(s) formed in both experiments is the complex of JEV 5'TR and JEV 3'TR.

\section{Determining the Affinity between JEV TRs}

With the above-mentioned relative binding affinity and direct evidence of interaction, we sought to quantify the affinity of the $5^{\prime}$ and $3^{\prime}$ TRs using MST. MST allows for the study of the interaction between a serially diluted target and fluorescently labeled ligand by measuring the change in fluorescent migration following excitation by an infrared laser (42). Using the difference between the "cold" and "hot" areas of the MST traces (Figure 5A), MST can determine the $K_{D}$ of the biomolecular interaction. Additionally, figure $5 A$ demonstrates that aggregation of the target was not observed (43) and that monomeric fractions were utilized in the assay. Our analysis indicates that the JEV TRs interact with a $K_{D}$ of $60 \pm 9 \mathrm{nM}$ (Figure 5B), which agrees with the previously reported $K_{D}$ of $32 \pm 1 \mathrm{nM}$ found in WNV using isothermal titration calorimetry (18), which is reasonably similar, validating our findings. Consequently, the binding affinity further validates our AUC experiment, performed with 200 nM concentrations of JEV TR RNA. To our knowledge, this is the first evidence of an RNA-RNA interaction characterized using MST. Next, we needed to validate whether the CS primarily drives this RNA-RNA interaction. We computationally predicted the RNA duplex secondary structure (Figure S3), showing numerous potential base-pairing sites across the interaction. To investigate whether the inclusion of the CS impacts binding affinity, we explored various fragments of the JEV TRs. JEV 3'UTR and JEV 5'TR, which include the CS, were then assayed and found to interact with a $K_{D}$ of $169 \pm 18 \mathrm{nM}$ (Figure $5 \mathrm{~B}$ ). As expected, the theoretically determined highest binding isolates of JEV (5'TR and 3'TR) are bound with a slightly higher affinity than the $3^{\prime}$ UTR and 5'TR but are within the same magnitude. When excluding this CS, as was the case with the assay of JEV 5'UTR, the interaction was considerably weaker with a $K_{D}$ of $23 \pm 6 \mu \mathrm{M}$. This change in affinity demonstrates that while the theoretical duplex interaction may have considerable base pairing, the primary driver of the interaction is the cyclization sequence. 
A

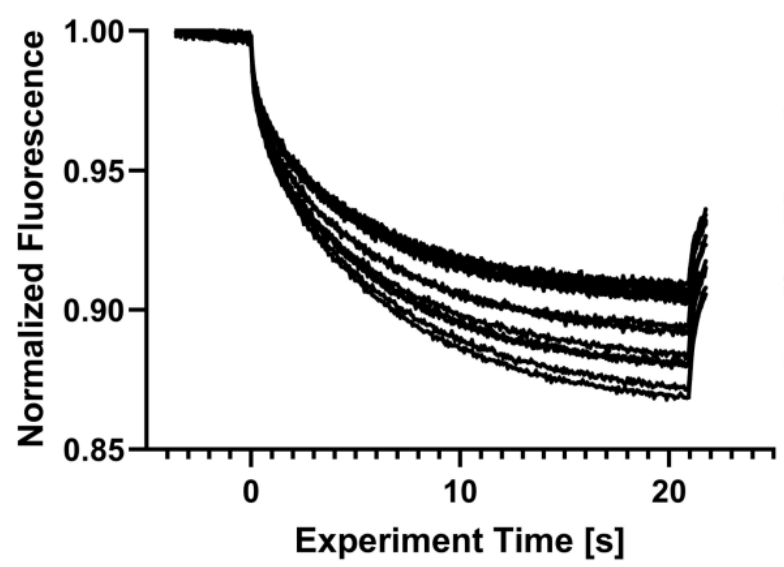

B

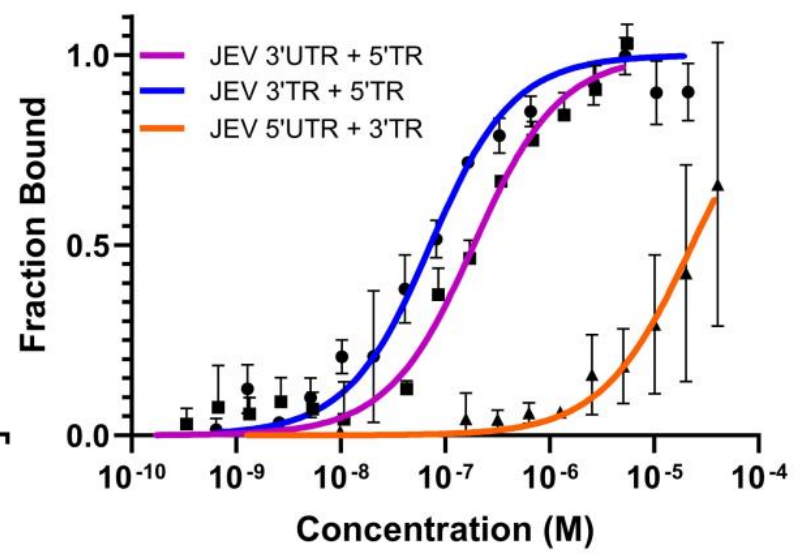

Figure 5. Affinity analysis of JEV TR RNA cyclization A) MST raw data traces for JEV 3'TR + 5'TR. The blue line represents the "cold" time, and the red line represents the "hot" region, and the difference between the two is used to calculate the $\Delta \mathrm{F}_{\text {norm }} \mathrm{B}$ ) Microscale thermophoresis measurements of different combinations of JEV TRs representing concentration vs. fraction bound.

\section{Kinetic and thermodynamic Studies of JEV 5' and 3' TRs}

As observed in Figure $3 \mathrm{~A}$ and Figure $4 \mathrm{~A}$, both TRs are likely to self-associate even though careful consideration was taken to avoid this. Therefore, we utilized computational analysis to explain the selfassociation of TRs potentially. While our predictions find potential homodimer interactions with similar thermodynamic stability as the heteroduplex (canonical CS), these homodimer interactions are quite extended and would require extensive refolding. Based on a kinetic analysis (Figure S2), we assume homodimers would form only short, less stable interactions, which would readily dissociate, favoring the more stable heterodimer $\left(3^{\prime}-5^{\prime}\right.$ complex) when mixed.

To help explain our MST observations, we compared the measured dissociation constants to the predicted interactions for the different TR and UTR constructs. We calculated binding free energies at 37 ${ }^{\circ} \mathrm{C}$ either from the measured dissociation constants or from predictions using RNAup (predictions are in parentheses). For the three constructs, the 5'TR and 3'TR, 5'TR and 3'UTR, and 5'UTR and 3'TR complex, we obtained $\Delta \mathrm{Gs}$ of $-10.2(-10.1) \mathrm{kcal} / \mathrm{mol},-9.6(-7.6) \mathrm{kcal} / \mathrm{mol}$, and $-6.6(-7.2) \mathrm{kcal} / \mathrm{mol}$, respectively. The more considerable discrepancy for the second construct (5'TR and 3'UTR) can be explained because the thermodynamic model predicts a refolding (compared to the known consensus structure) when the longer $3^{\prime} U T R$ is used occluding the CS.

We also investigated potential additional interaction sites to the known CS. The most promising is a kinetically favourable interaction corresponding to the known upstream AUG region (UAR) (44) with predicted stability of $-3.6 \mathrm{kcal} / \mathrm{mol}$. This interaction could further stabilize the CS interaction but is not strong enough to explain the duplex formation on its own. 


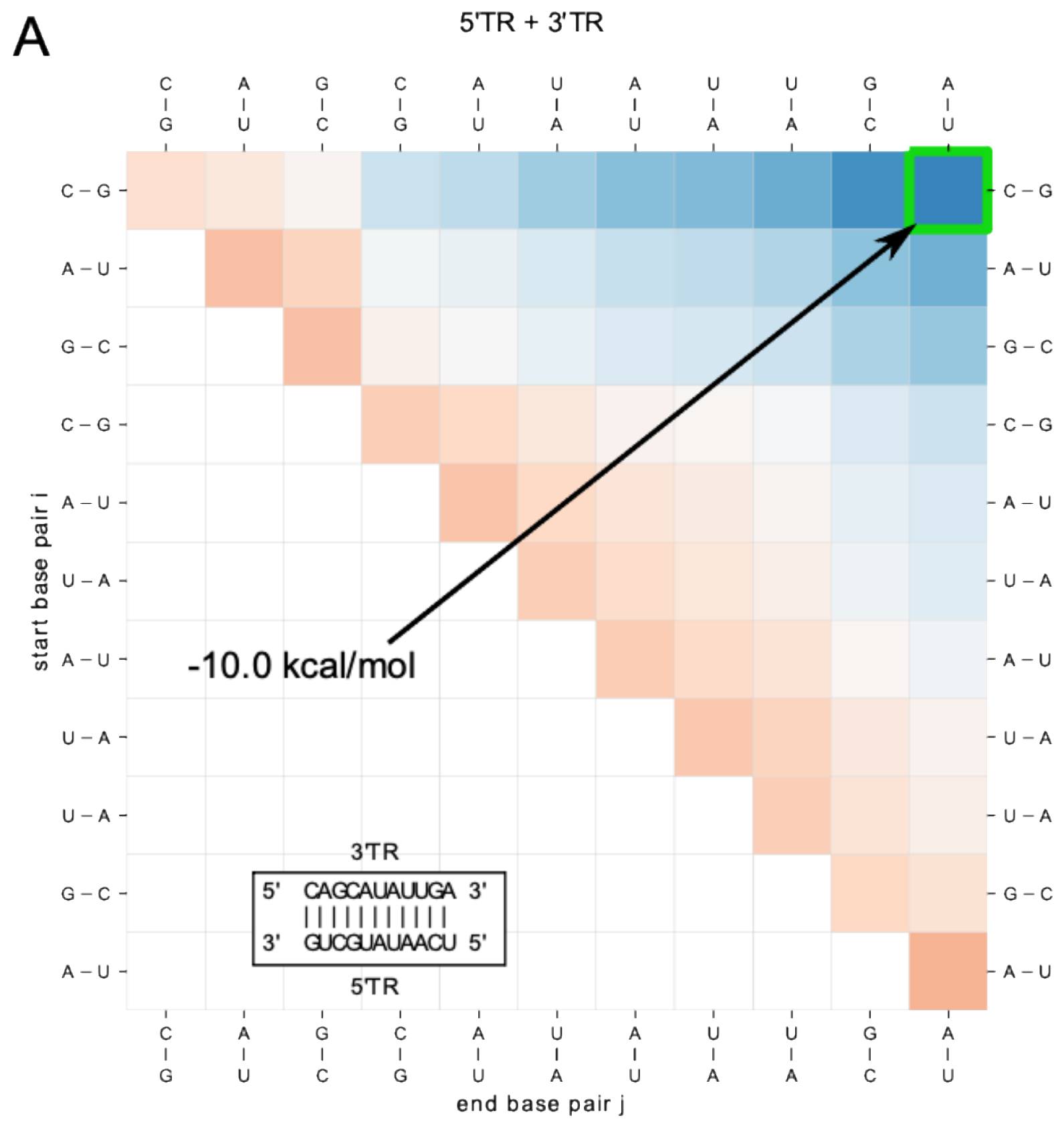

Figure 6. Energy landscape of the predicted 3'TR and 5'TR CS interaction. The energy landscape represents all possible substructures that can occur along a direct path from a single base pair interaction to the entire CS interaction.

\section{Conclusion}

Our study demonstrates that combining computational and biophysical approaches can provide detailed insights into RNA-RNA interactions that govern many fundamental biological processes. Our SEC- 
MALS, AUC, and MST data also suggest, for the first time, that these techniques can be used to study RNARNA interactions in solution. We also demonstrate that computational analysis such as kinetic landscapes can provide vital evidence for predicting RNA-RNA interactions and even sites of interactions. Additionally, our binding affinity data presents that the CS is the primary driver of terminal region interaction, with a nanomolar affinity. This binding affinity is critical to developing potential inhibitory therapeutics targeting the cyclization sequence. Now that the binding affinity is well characterized, a benchmark affinity should be carefully considered when discussing therapeutic design. Flaviviral cyclization conservation should also be considered when developing therapeutics as a potential for multi-virus inhibition.

\section{Data availability}

JEV isolates for computational analysis: We selected complete JEV isolates (including 3' and 5' UTR) from NCBI GenBank and used the first 225 nts (5'TR) and the last 221 nts (3'TR) to perform interaction predictions. The corresponding accession numbers can be found in the 'JEV.txt' supplemental file.

\section{Funding and additional information}

T.M. is supported by a Natural Sciences and Engineering Research Council (NSERC) PGS-D award. S.M.P is supported by the NSERC Discovery grant awarded to T.R.P., and C.R.N. was supported by Alberta Innovates Graduate student award. M.W. is supported by the Austrian Science Fund (FWF I-2874-N28, DK RNA Biology, F 80 RNAdeco awarded to ILH). This research was funded by the NSERC Discovery grant, RGPIN-2017-04003, to T.R.P., T.R.P. is a Canada Research Chair in RNA and Protein Biophysics. Infrastructure support to T.R.P. was provided by the Canada Foundation for Innovation and NSERC RTI Grants. This work was supported by the Canada 150 Research Chairs program (C150-2017-00015, BD), the Canada Foundation for Innovation (CFI-37589, BD), the National Institutes of Health (1R01GM120600, $B D$ ), and the NSERC Discovery grant (RGPIN-2019-05637, BD). UltraScan supercomputer calculations supported NSF/XSEDE grant TG-MCB070039N (BD). The NSERC supports A.H. through a scholarship grant.

\section{Conflict of Interest Disclosure}

The author(s) declare(s) that there is no conflict of interest. 


\section{References}

1. Heffelfinger, J.D., Li, X., Batmunkh, N., Grabovac, V., Diorditsa, S., Liyanage, J.B., Pattamadilok, S., Bahl, S., Vannice, K.S., Hyde, T.B. et al. (2017) Japanese Encephalitis Surveillance and Immunization - Asia and Western Pacific Regions, 2016. MMWR Morb Mortal Wkly Rep, 66, 579583.

2. Campbell, G.L., Hills, S.L., Fischer, M., Jacobson, J.A., Hoke, C.H., Hombach, J.M., Marfin, A.A., Solomon, T., Tsai, T.F., Tsu, V.D. et al. (2011) Estimated global incidence of Japanese encephalitis: a systematic review. Bull World Health Organ, 89, 766-774, 774a-774e.

3. Wang, Q.-Y. and Shi, P.-Y. (2015) Flavivirus Entry Inhibitors. ACS Infectious Diseases, 1, 428-434.

4. Huang, Y.J., Higgs, S., Horne, K.M. and Vanlandingham, D.L. (2014) Flavivirus-mosquito interactions. Viruses, 6, 4703-4730.

5. Tjaden, N.B., Caminade, C., Beierkuhnlein, C. and Thomas, S.M. (2018) Mosquito-Borne Diseases: Advances in Modelling Climate-Change Impacts. Trends in Parasitology, 34, 227-245.

6. Lindenbach, B., Thiel, H.J. and Rice, C.M. (2007) Flaviviridae: The viruses and their replication. Fields Virology, 1101-1151.

7. Ng, W.C., Soto-Acosta, R., Bradrick, S.S., Garcia-Blanco, M.A. and Ooi, E.E. (2017) The $5^{\prime}$ and $3^{\prime}$ Untranslated Regions of the Flaviviral Genome. Viruses, 9, 137.

8. Bollati, M., Alvarez, K., Assenberg, R., Baronti, C., Canard, B., Cook, S., Coutard, B., Decroly, E., de Lamballerie, X., Gould, E.A. et al. (2010) Structure and functionality in flavivirus NS-proteins: perspectives for drug design. Antiviral research, 87, 125-148.

9. Mandl, C.W., Holzmann, H., Kunz, C. and Heinz, F.X. (1993) Complete Genomic Sequence of Powassan Virus: Evaluation of Genetic Elements in Tick-Borne versus Mosquito-Borne Flaviviruses. Virology, 194, 173-184.

10. Filomatori, C.V., Lodeiro, M.F., Alvarez, D.E., Samsa, M.M., Pietrasanta, L. and Gamarnik, A.V. (2006) A 5' RNA element promotes dengue virus RNA synthesis on a circular genome. Genes Dev, 20, 2238-2249.

11. You, S. and Padmanabhan, R. (1999) A Novel in Vitro Replication System for Dengue Virus: INITIATION OF RNA SYNTHESIS AT THE 3'-END OF EXOGENOUS VIRAL RNA TEMPLATES REQUIRES 5'- AND 3'-TERMINAL COMPLEMENTARY SEQUENCE MOTIFS OF THE VIRAL RNA*. Journal of Biological Chemistry, 274, 33714-33722.

12. Zhang, B., Dong, H., Stein, D.A., Iversen, P.L. and Shi, P.-Y. (2008) West Nile virus genome cyclization and RNA replication require two pairs of long-distance RNA interactions. Virology, 373, 1-13.

13. Alvarez, D.E., Lodeiro, M.F., Ludueña, S.J., Pietrasanta, L.I. and Gamarnik, A.V. (2005) Long-range RNA-RNA interactions circularize the dengue virus genome. Journal of virology, 79, 6631-6643.

14. Hahn, C.S., Hahn, Y.S., Rice, C.M., Lee, E., Dalgarno, L., Strauss, E.G. and Strauss, J.H. (1987) Conserved elements in the $3^{\prime}$ untranslated region of flavivirus RNAs and potential cyclization sequences. J Mol Biol, 198, 33-41.

15. Meier-Stephenson, V., Mrozowich, T., Pham, M. and Patel, T.R. (2018) DEAD-box helicases: the Yin and Yang roles in viral infections. Biotechnology \& genetic engineering reviews, 34, 3-32.

16. Nelson, C., Mrozowich, T., Gemmill, D.L., Park, S.M. and Patel, T.R. (2021) Human DDX3X Unwinds Japanese Encephalitis and Zika Viral 5' Terminal Regions. Int J Mol Sci, 22, 413.

17. Men, R., Bray, M., Clark, D., Chanock, R.M. and Lai, C.J. (1996) Dengue type 4 virus mutants containing deletions in the 3 ' non-coding region of the RNA genome: analysis of growth restriction in cell culture and altered viremia pattern and immunogenicity in rhesus monkeys. Journal of virology, 70, 3930-3937. 
18. Deo, S., Patel, T.R., Chojnowski, G., Koul, A., Dzananovic, E., McEleney, K., Bujnicki, J.M. and McKenna, S.A. (2015) Characterization of the termini of the West Nile virus genome and their interactions with the small isoform of the 2' 5'-oligoadenylate synthetase family. Journal of structural biology, 190, 236-249.

19. Bredenbeek, P.J., Kooi, E.A., Lindenbach, B., Huijkman, N., Rice, C.M. and Spaan, W.J.M. (2003) A stable full-length yellow fever virus cDNA clone and the role of conserved RNA elements in flavivirus replication. Journal of General Virology, 84, 1261-1268.

20. Khromykh Alexander, A., Meka, H., Guyatt Kimberley, J. and Westaway Edwin, G. (2001) Essential Role of Cyclization Sequences in Flavivirus RNA Replication. Journal of virology, 75, 6719-6728.

21. Kofler, R.M., Hoenninger, V.M., Thurner, C. and Mandl, C.W. (2006) Functional analysis of the tickborne encephalitis virus cyclization elements indicates major differences between mosquitoborne and tick-borne flaviviruses. Journal of virology, 80, 4099-4113.

22. Corver, J., Lenches, E., Smith, K., Robison, R.A., Sando, T., Strauss, E.G. and Strauss, J.H. (2003) Fine mapping of a cis-acting sequence element in yellow fever virus RNA that is required for RNA replication and cyclization. Journal of virology, 77, 2265-2270.

23. Lo, M.K., Tilgner, M., Bernard, K.A. and Shi, P.Y. (2003) Functional analysis of mosquito-borne flavivirus conserved sequence elements within 3' untranslated region of West Nile virus by use of a reporting replicon that differentiates between viral translation and RNA replication. Journal of virology, 77, 10004-10014.

24. Thurner, C., Witwer, C., Hofacker, I.L. and Stadler, P.F. (2004) Conserved RNA secondary structures in Flaviviridae genomes. Journal of General Virology, 85, 1113-1124.

25. Mann, M., Wright, P.R. and Backofen, R. (2017) IntaRNA 2.0: enhanced and customizable prediction of RNA-RNA interactions. Nucleic acids research, 45, W435-W439.

26. Busch, A., Richter, A.S. and Backofen, R. (2008) IntaRNA: efficient prediction of bacterial sRNA targets incorporating target site accessibility and seed regions. Bioinformatics, 24, 2849-2856.

27. Mückstein, U., Tafer, H., Hackermüller, J., Bernhart, S.H., Stadler, P.F. and Hofacker, I.L. (2006) Thermodynamics of RNA-RNA binding. Bioinformatics, 22, 1177-1182.

28. Hofacker, I.L., Fontana, W., Stadler, P.F., Bonhoeffer, L.S., Tacker, M. and Schuster, P. (1994) Fast folding and comparison of RNA secondary structures. Monatshefte für Chemie / Chemical Monthly, 125, 167-188.

29. Bernhart, S.H., Tafer, H., Mückstein, U., Flamm, C., Stadler, P.F. and Hofacker, I.L. (2006) Partition function and base pairing probabilities of RNA heterodimers. Algorithms for Molecular Biology, 1, 3.

30. Bernhart, S.H., Hofacker, I.L., Will, S., Gruber, A.R. and Stadler, P.F. (2008) RNAalifold: improved consensus structure prediction for RNA alignments. BMC Bioinformatics, 9, 474.

31. Lorenz, R., Bernhart, S.H., Höner zu Siederdissen, C., Tafer, H., Flamm, C., Stadler, P.F. and Hofacker, I.L. (2011) ViennaRNA Package 2.0. Algorithms for Molecular Biology, 6, 26.

32. Will, S., Reiche, K., Hofacker, I.L., Stadler, P.F. and Backofen, R. (2007) Inferring Noncoding RNA Families and Classes by Means of Genome-Scale Structure-Based Clustering. PLOS Computational Biology, 3, e65.

33. Pam Wang, R.A., Michelle Chen, Kristine Legaspi. (2020), Wyatt Technologies, pp. 1-4.

34. Demeler, B. and Gorbet, G.E. (2016) In Uchiyama, S., Arisaka, F., Stafford, W. F. and Laue, T. (eds.), Analytical Ultracentrifugation: Instrumentation, Software, and Applications. Springer Japan, Tokyo, pp. 119-143.

35. Demeler, B. and van Holde, K.E. (2004) Sedimentation velocity analysis of highly heterogeneous systems. Analytical Biochemistry, 335, 279-288.

36. Ochsenreiter, R., Hofacker, I.L. and Wolfinger, M.T. (2019) Functional RNA Structures in the 3'UTR of Tick-Borne, Insect-Specific and No-Known-Vector Flaviviruses. Viruses, 11, 298. 
37. Michael T. Wolfinger, R.O., Ivo L. Hofacker. (2021) In Marz, D. F. a. M. (ed.), Virus Bioinformatics. 1 ed. Chapman and Hall/CRC, pp. 36.

38. Nelson, C.R., Mrozowich, T., Park, S.M., D'souza, S., Henrickson, A., Vigar, J.R.J., Wieden, H.-J., Owens, R.J., Demeler, B. and Patel, T.R. (2021) Human DDX17 Unwinds Rift Valley Fever Virus NonCoding RNAs. Int J Mol Sci, 22, 54.

39. Mrozowich, T., Henrickson, A., Demeler, B. and Patel, T.R. (2020) Nanoscale Structure Determination of Murray Valley Encephalitis and Powassan Virus Non-Coding RNAs. Viruses, 12, 190.

40. Wyatt, P.J. (1993) Light scattering and the absolute characterization of macromolecules. Analytica Chimica Acta, 272, 1-40.

41. Patel, T.R., Winzor, D.J. and Scott, D.J. (2016) Analytical ultracentrifugation: A versatile tool for the characterisation of macromolecular complexes in solution. Methods, 95, 55-61.

42. Wienken, C.J., Baaske, P., Rothbauer, U., Braun, D. and Duhr, S. (2010) Protein-binding assays in biological liquids using microscale thermophoresis. Nature Communications, 1, 100.

43. Mrozowich, T., MeierStephenson, V. and Patel, T.R. (2019) Microscale thermophoresis: warming up to a new biomolecular interaction technique. The Biochemist, 41, 8-12.

44. Friebe, P., Shi, P.-Y. and Harris, E. (2011) The 5' and 3' downstream AUG region elements are required for mosquito-borne flavivirus RNA replication. Journal of virology, 85, 1900-1905. 